\title{
Implementation of the Feed and Swaddle Technique as a Non-Pharmacological Strategy to Conduct Brain Magnetic Resonance Imaging in Very Low Birth Weight Infants
}

\author{
Yeong Myong Yoo, $\mathrm{MD}^{1}$, Ji Eun Park, MD, $\mathrm{PhD}^{2}$, Moon Sung Park, MD, $\mathrm{PhD}^{3}$, and Jang Hoon Lee, MD, $\mathrm{PhD}^{3}$ \\ ${ }^{1}$ Department of Pediatrics, Wonju Severance Christian Hospital, Yonsei University Wonju College of Medicine, Wonju, Korea \\ Departments of ${ }^{2}$ Radiology and ${ }^{3}$ Pediatrics, Ajou University Hospital, Ajou University School of Medicine, Suwon, Korea
}

\section{ABSTRACT}

Purpose: Magnetic resonance imaging (MRI) is a useful tool for evaluating brain injury and maturation in preterm infants and often requires sedation to acquire images of sufficient quality. Infant sedation is often associated with adverse events, despite extreme precautions. In this study, the swaddling technique was investigated as an alternative non-pharmacological strategy to obtain brain MRIs of sufficient quality.

Methods: We applied the feed and swaddle technique during routine brain MRI as a quality improvement project and compared its morbidity with that of sedation in a historic age-matched group. Seventy-nine very low birth weight infants in the neonatal intensive care unit of Ajou University Hospital (Suwon, Korea) were enrolled. Thirty-two (40.5\%) infants were in the feed and swaddling group, and 47 (59.5\%) were in the sedation group.

Results: The morbidity associated with the cardiopulmonary system (swaddling group vs. sedation group: $53.13 \%$ [n=17] vs. $63.83 \%[\mathrm{n}=30], P=0.723)$ and central nervous system $(40.63 \%$ [n=13] vs. $29.79 \%[\mathrm{n}=14], P=0.217)$ were not significantly different between groups. The MRI failure rate was not significantly different (swaddling group vs. sedation group: $12.5 \%[\mathrm{n}=4]$ vs. $4.3 \%[\mathrm{n}=2], P=0.174$ ). The MRI scanning time was longer in the swaddling group than in the sedation group ( $76.5 \pm 20.3$ minutes vs. $61.5 \pm 13.6$ minutes, $P=0.001$ ). Cardiopulmonary adverse events were significantly less common in the swaddling group than in the sedation group $(3.13 \%[\mathrm{n}=1]$ vs. $34.04 \%[\mathrm{n}=16], P=0.002)$.

Conclusion: The success rate of MRI was comparable between the swaddling technique and sedation. Furthermore, despite the drawback of prolonged scan time, cardiopulmonary adverse events are fewer with swaddling than with sedative agents. Therefore, swaddling can be an alternative to sedation or anesthesia when performing neonatal MRI scans.

Key Words: Swaddling; Sedation; Infant, very low birth weight; Magnetic resonance imaging
Received: 7 May 2021

Revised: 21 June 2021

Accepted: 28 June 2021

Correspondence to: Jang Hoon Lee, $\mathrm{MD}, \mathrm{PhD}$

Department of Pediatrics, Ajou Uni versity School of Medicine, 164 World cup-ro, Yeongtong-gu, Suwon 16499, Korea

Tel: +82-31-219-5167

Fax: +82-31-219-5169

E-mail: neopedlee@aumc.ac.kr

Copyright(c)

By Korean Society of Neonatology.

This is an Open-Access article distributed under the terms of the Creative Commons At tribution Non-Commercial License (http:// creativecommons.org/licenses/by-nc/4.0), which permits unrestricted non-commercial use, distribution, and reproduction in any medium, provided the original work is properly cited. 


\section{INTRODUCTION}

The risk of neurodevelopmental and psychobehavioral pro blems are significantly greater in preterm infants, especially in very low birth weight infants (VLBWIs) ${ }^{1,2)}$. Cranial ultrasound is traditionally the modality of choice for screening intracranial lesions in preterm infants. At near-term or before discharging an infant from a neonatal intensive care unit (NICU), brain magnetic resonance imaging (MRI) is often recommended to determine an early prognosis and as a potential guide for early intervention $^{3)}$. However, MRI is sensitive to motion artifacts; therefore, immobilization during the test is essential to obtain images of sufficient quality in infants. Several strategies have been postulated for immobilization, such as general anesthesia, sedation, and non-pharmacological methods including as the feed and swaddle technique ${ }^{4)}$. Non-pharmacological interventions, such as swaddling, non-nutritive suckling, administering breast milk, or providing skin-to-skin contact with the mother, may be alternatives ${ }^{5}$. Common terms for a nonpharmacologic approach are "feed and sleep," "feed and wrap," and "feed and swaddle"6). General anesthesia or sedation has been a common practice in pediatric MRI procedures ${ }^{7)}$. Many sedatives and anesthetics are potent modulators of important morphogenetic events during brain development ${ }^{8,9)}$ and are possible risk factors for developmental delay, learning disability, and attention deficithypersensitivity disorder ${ }^{10-12)}$. Moreover, general anesthesia or sedation may cause serious adverse events, such as airway obstruction, oxygen desaturation, hemodynamic complications, and cardiac arrest, especially in preterm infants ${ }^{13,14)}$. Thus, many institutes have described non-pharmacological techniques to immobilize infants during MRI ${ }^{15,16)}$. However, many institutes have not implemented these guidelines.

A standardized MRI guideline, including the use of sedation and safe transport, has been implemented in our NICU since 2010. In 2018, our multidisciplinary team implemented a quality improvement(QI) project to minimize the use of sedatives during MRI in the NICU. In this article, we describe our QI experience with sedation-free brain MRI studies with regard to safety and efficiency.

\section{MATERIALS AND METHODS}

This study was a retrospective study targeting VLBWIs who underwent MRI in the NICU of Ajou University Hospital (Suwon, Korea) from May 2017 to December 2018. The patients were divided into the sedation group (treated between May 2017 and February 2018) and the feed and swaddle technique group (treated from March 2018 to December 2018). The feed and swaddle technique was implemented in 2018 for the purpose of the Joint Commission International accreditation of the QI project.

From May 2017 to February 2018 at our institute, infant brain MRI was conducted with conscious sedation and an immobilizer (MedVac vacuum splint, Kohlbrat \& Bunz Co. Ltd., Radstadt, Austria). Two months before initiating the QI project, we reviewed the literature and developed a protocol for the feed and swaddle guidelines for our institute.

\section{Patients}

We investigated the data of VLBWIs who received swaddling or sedatives (e.g., chloral hydrates and/or benzodiazepines) for brain MRI in the NICU of Ajou University Hospital. The inclusion criteria for the sedation and swaddling groups differed only in the implementation of the QI policy: all patients who underwent brain MRI before the implementation of the QI process were in the sedation group, and those who underwent brain MRI after the QI process were in the swaddling group.

Conventional brain MRI with T1-weighted images, T2-weighted images, and fluid-attenuated inversion recovery images without contrast were obtained from all patients. Data were derived from the medical records at Ajou University Hospital.

Patient demographic data, such as gestational age (GA), birth weight (BW), weight at MRI, and comorbidities, were collected. The MRI failure rate, scan time, and adverse events during and after the procedure were recorded.

\section{Equipment and process}

A MedVac vacuum splint was used as the customized immobilizer (Figure 1). The size of the MedVac vacuum splint, number of divisions inside the MedVac vacuum splint, and number of filled beads in the MedVac vacuum splint can be customized when ordering the device. The upper part immobilized the head, and the lower part immobilized the trunk. After adjusting the shape, the MedVac vacuum splint hardened and did not collapse when the internal air was removed through the exhaust aspirator. The customized MedVac vacuum splint was designed to be filled with air to fit the patient's body shape after wrapping the chest 


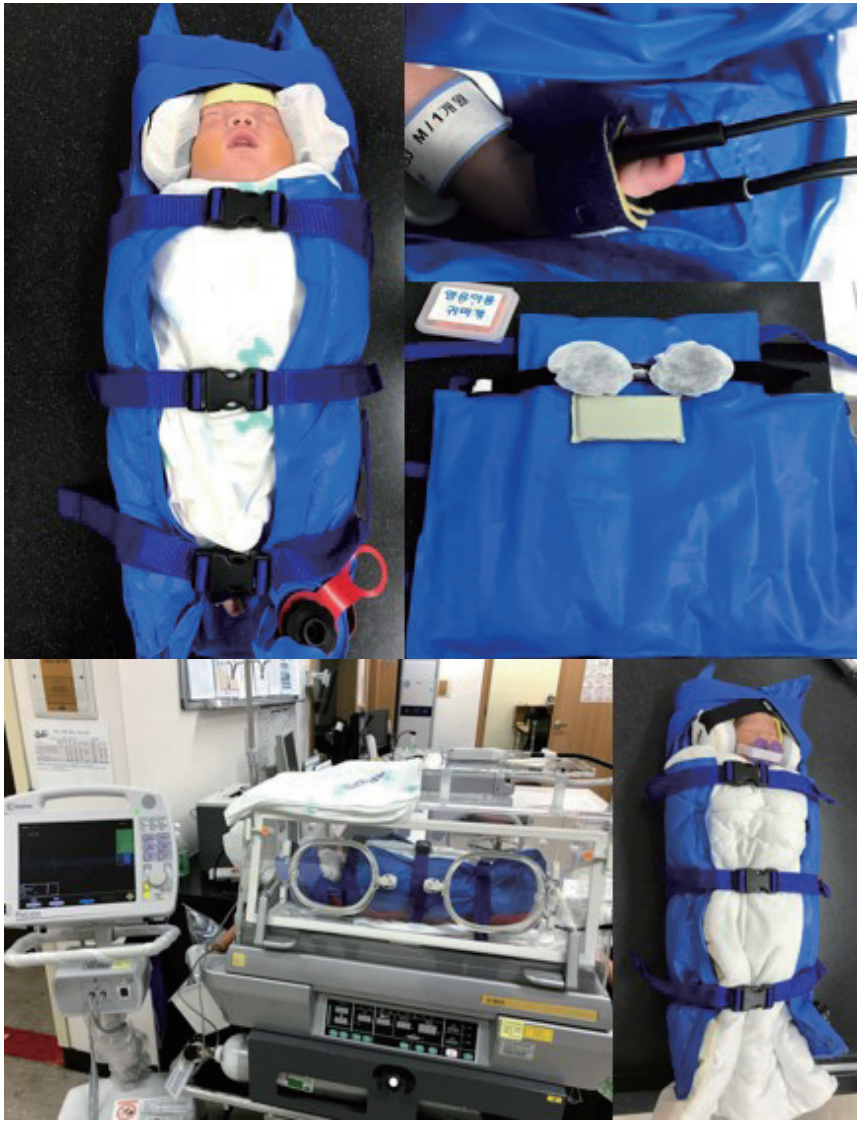

Figure 1. The MedVac vacuum splint (Kohlbrat \& Bunz Co. Ltd.) and a patient connected to monitors in the magnetic resonance imaging room.

and abdomen, and there were no cases of dyspnea or vomiting among the patients.

In the swaddling group, the infants were not fasted and did not have feeding restrictions before MRI. Approximately 30 minutes before the MRI, the patients were fed formula milk or breast milk as they normally ingested in the NICU. If necessary, a pacifier was used. Before feeding, attachments on the patient's body were removed, the diaper was replaced, and the patient was wrapped in a blanket to minimize disturbance after sleep. When wrapping the patient in a blanket, one foot remained outside the blanket to facilitate patient identification and attach the monitoring sensor. After feeding, ear plugs were attached to the patient's ears with tape. The prepared infants were transferred to a transport incubator, and oxygen saturation monitoring was continued in the MRI room. One physician's assistant dedicated to the newborn was responsible for patient transport and monitoring.

After arriving in the MRI room, the infant was placed on the immobilizer (i.e., the MedVac vacuum splint) and earmuffs were placed for noise prevention and head fixation. The sensor was then replaced with an MRI sensor attached to the foot of the patient and fixed using the vacuum splint.

Owing to the nature of gastrointestinal function in premature infants, feeding restrictions can significantly reduce gastric motility; therefore, the preparation of the sedation group did not involve fasting or any feeding restrictions. Moreover, before the QI project, it was difficult to predict the MRI scan time through collaboration with the department of radiology; therefore, scheduled feeding was routinely performed. However, approximately 20 to 30 minutes before the test, a gastric tube was noninvasively used to remove any residual milk in the patient's stomach. After removing the gastric contents, all patients in the sedation group were orally administered $50 \mathrm{mg} / \mathrm{kg}$ chloral hydrate in the NICU. Oxygen saturation and heart rate were monitored after chloral hydrate administration. Before transferring the infant, an additional $0.1 \mathrm{mg} / \mathrm{kg}$ intravenous benzodiazepine (i.e., midazolam) was prepared, which could be used for patient sedation.

Before the MRI, the patients were evaluated to determine whether they remained asleep. Patients confirmed to be asleep underwent MRI, and those who awoke from sleep were provided a quiet environment without additional medication and then underwent MRI when movement decreased. During MRI, the prepared midazolam was intravenously injected to induce sleep if a patient's movement interfered with imaging progression. Patients who did not enter sleep after two midazolam doses were removed from the study, and the test was recorded as a failure.

Collected data were analyzed using SPSS version 25.0 (IBM Corp., Armonk, NY, USA). Differences between the swaddling and sedation groups, based on GA and BW, were compared according to the type of variable. Categorical data are reported as numbers and percentages for proportions. These data were compared using the chi-square test or Fisher's exact test. Continuous data are reported as the mean \pm standard deviation. These data were compared using Student's $t$-test or the Mann-Whitney $U$-test. Statistical significance was set at $P<0.05$.

\section{Adverse events and morbidity}

Adverse events caused by the imaging process were evaluated based on changes in vital signs at the time of and after imaging. The types of adverse events at the time of MRI were classified using three criteria: decreased peripheral oxygen saturation, changes in heart rate, and prearrest status. A decrease in oxygen saturation was defined as a decrease in peripheral oxygen 
saturation to less than $90 \%$ on the monitor, accompanied by breathing cessation for more than 20 seconds. A change in heart rate was defined as an increase in the heart rate above 180 beats/ min or a decrease in the heart rate below 80 beats/min for more than 15 seconds without any symptoms of agitation or change in oxygen saturation. Prearrest status was defined as a decrease in oxygen saturation below $90 \%$ and a decrease in the heart rate below 60 beats/min; adverse events after the imaging process were defined using the same criteria and occurring within 24 hours after the test ${ }^{17}$.

Comorbidity, which may affect the adverse events of sedation and swallowing, was classified as cardiopulmonary system comorbidity or central nervous system comorbidity. Cardiopulmonary system morbidities included bronchopulmo nary dysplasia, congenital heart disease, oxygen dependence at the time of MRI, and symptomatic patent ductus arteriosus (excluding asymptomatic patent ductus arteriosus), atrial septal defect, and patent foramen ovale. Central nervous system morbidities included cerebral malformation, intracranial he morrhage, intraventricular hemorrhage of grade $\geq$ III, and periventricular leukomalacia ${ }^{18)}$.

\section{Success rate/running time/imaging quality}

The success or failure of MRI was determined retrospectively by obtaining a qualified image that could be read by pediatric radiologists. The MRI scan time was the duration between when the patient was placed on the scanner table and when the patient exited the scanner after completing all planned imaging. If the patient moved during the scan and needed additional medication or adjustment, this time was included in the scan time. The MRI-to-feeding time was defined as the duration between the time when the patient left the MRI room and the first scheduled feeding. Overall, the MRI image quality was assessed retrospectively and independently by two board-certified pediatric radiologists. The two reviewers were blinded to the patient information

\section{RESULTS}

\section{Patients' characteristics}

Among the enrolled infants $(n=79), 40.5 \%(n=32)$ were in the swaddling group, $59.5 \%(\mathrm{n}=47)$ were in the sedation group, and $54.4 \%(n=43)$ were boys and $45.6 \%(n=36)$ were girls. The mean GA and BW at birth of the total population were $29^{+4} \pm 5^{+3}$ weeks and $1,176.1 \pm 253.1 \mathrm{~g}$, respectively. The postconceptional age and body weight when undergoing MRI were $37^{+4} \pm 5^{+3}$ weeks and 2,327.0 $\pm 528.7 \mathrm{~g}$, respectively. When undergoing MRI, most infants had a BW of $1,700.0$ to 2,200.0 $\mathrm{g}(\mathrm{n}=39,49.3 \%)$, followed by infants weighing $2,200.0$ to $2,700.0 \mathrm{~g}(\mathrm{n}=23,29.2 \%)$. With regard to the corrected GA at the time of MRI, the highest proportion of patients ( $49.4 \%, \mathrm{n}=39$ ) was 36 to 39 weeks old. The mean time from birth to MRI was $56.0 \pm 30.4$ days.

There was no statistically significant difference in sex ratio (53.13\% in men [swaddling] vs. $55.32 \%$ in males [sedation], $P=0.848)$. In addition, no statistical significance was found for whether the patient was a multipara child (53.13\% in multipara [swaddling] vs. 31.91\% in multipara [sedation], $P=0.059$ ).

No significant differences were observed in GA at birth $\left(29^{+3} \pm 4^{+1}\right.$ [swaddling] vs. $29^{+6} \pm 5^{+1}$ [sedation], $\left.P=0.439\right)$ or corrected GA at the time of MRI $\left(37^{+4} \pm 2^{+3}\right.$ vs. $\left.37^{+6} \pm 3^{+1}, P=0.649\right)$. The mean body weight at birth (swaddling group vs. sedation group: $1,188.9 \pm 246.4 \mathrm{~g}$ vs. $1,167.4 \pm 259.9 \mathrm{~g}, P=0.714)$ and at the time of MRI $(2,439.7 \pm 484.4$ g vs. $2,250.3 \pm 548.7 \mathrm{~g}, P=0.119)$ were similar (Table 1).

Table 1. Patients' Characteristics Based on Whether the Infant Is or Is Not Sedated

\begin{tabular}{lccc}
\hline \multirow{2}{*}{ Characteristic } & \multicolumn{2}{c}{ Group } & P-value \\
\cline { 2 - 3 } & Swaddling group (n=32) & Sedation group (n=47) \\
\hline Male sex & $17(53.13)$ & $26(55.32)$ & $15(31.91)$ \\
Multipara & $17(53.13)$ & $29^{+6} \pm 5^{+1}$ & 0.848 \\
GA at birth (wk) & $29^{+3} \pm 4^{+1}$ & $37^{+6} \pm 3^{+1}$ & 0.439 \\
Corrected GA at MRI (wk) & $37^{+4} \pm 2^{+3}$ & $1,167.4 \pm 259.9$ & 0.649 \\
Mean BW at birth (g) & $1,188.9 \pm 246.4$ & $2,250.3 \pm 548.7$ & 0.714 \\
Mean BW at the time of MRI (g) & $2,439.7 \pm 484.4$ & & 0.119 \\
\hline
\end{tabular}

Values are expressed as number (\%) or mean \pm standard deviation.

Abbreviations: GA, gestational age; MRI, magnetic resonance imaging; BW, birth weight. 


\section{Morbidity}

Morbidities associated with the cardiopulmonary system were not significantly different between the swaddling and sedation groups (53.13\% [n=17] vs. $63.83 \%$ [ $\mathrm{n}=30], P=0.723)$, bronchopulmonary dysplasia $(P=0.621)$, congenital heart disease $(P=0.517)$, oxygen dependency $(P=0.334)$, and symptomatic patent ductus arteriosus $(P=0.798)$ (Table 2). No significant differences were observed in central nervous system morbidities between the swaddling and sedation groups $(40.63 \%[\mathrm{n}=13]$ vs. $29.79 \%$ [n=14], $P=0.217)$, cerebral malformations $(P=0.855)$, intracranial hemorrhage $(P=0.045)$, intraventricular hemorrhage $(P=0.796)$, and periventricular leukomalacia $(P=0.517)$ (Table 2$)$.

\section{Adverse events during imaging process}

Adverse events during MRI in the swaddling and sedation groups showed decreased oxygen saturation $(3.13 \%[\mathrm{n}=1]$ vs. $4.26 \%[\mathrm{n}=2], P=0.796)$ and change in heart rate $(0.00 \%[\mathrm{n}=0]$ vs. $14.89 \%[\mathrm{n}=7], P=0.022)$. Delayed adverse events occurring within 24 hours after MRI in the swaddling group versus the sedation group were decreased oxygen saturation with repeated apnea $(0.00 \%$ [n=0] vs. $12.77 \%[\mathrm{n}=6], P=0.036)$ and prearrest status $(0.00 \%[\mathrm{n}=0]$ vs. $2.13 \%[\mathrm{n}=1], P=0.406)$.

Cardiopulmonary adverse events occurring with the MRI test were significantly lower in the swaddling group than in the sedation group $(3.13 \%[\mathrm{n}=1]$ vs. $34.04 \%[\mathrm{n}=16], P=0.002)$. In

Table 2. Comorbidities Associated with Adverse Events at the Time of Magnetic Resonance Imaging

\begin{tabular}{lccc}
\hline Variable & Swaddling group $(\mathrm{n}=32)$ & Sedation group $(\mathrm{n}=47)$ & $P$-value \\
\hline Morbidity associated with CPS & $17(53.13)$ & $30(63.83)$ & $11(23.40)$ \\
BPD & $6(18.75)$ & $3(6.38)$ & 0.723 \\
CHD & $1(3.13)$ & $4(8.51)$ & 0.621 \\
Oxygen dependency & $1(3.13)$ & $12(25.53)$ & 0.517 \\
Symptomatic PDA & $9(28.13)$ & $14(29.79)$ & 0.334 \\
Morbidity associated with CNS & $13(40.63)$ & $4(8.51)$ & 0.217 \\
Cerebral malformation & $3(9.38)$ & $2(4.26)$ & 0.045 \\
ICH & $8(25.00)$ & $3(6.38)$ & 0.796 \\
IVH (grade III or higher) & $1(3.13)$ & $1(3.13)$ & 0.517 \\
PVL & &
\end{tabular}

Values are expressed as number (\%).

Abbreviations: CPS, cardiopulmonary system; BPD, bronchopulmonary dysplasia; CHD, congenital heart disease; PDA, patent ductus arteriosus; CNS, central nervous system; ICH, intracranial hemorrhage; IVH, intraventricular hemorrhage; PVL, periventricular leukomalacia.

Table 3. Magnetic Resonance Imaging Outcome and Cardiopulmonary Adverse Events Associated with the Imaging Process

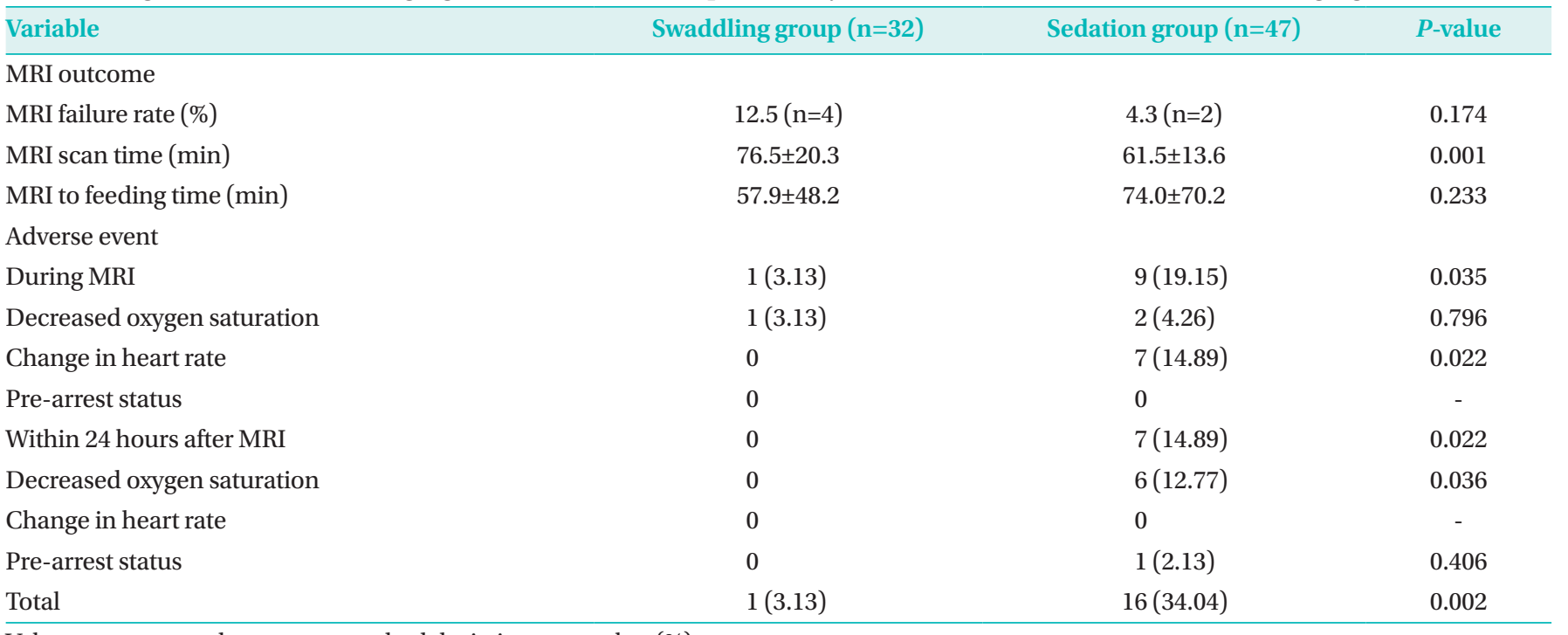

Values are expressed as mean \pm standard deviation or number (\%).

Abbreviation: MRI, magnetic resonance imaging. 
addition, a comparison of adverse events within 24 hours and at the time of scanning revealed that the sedation group had significantly delayed respiratory inhibition, compared to the swaddling group ( $14.89 \%[\mathrm{n}=7]$ vs. $0.00 \%[\mathrm{n}=0], P=0.022$ ) (Table 3).

\section{Success rate/running time}

No significant difference was observed in the MRI failure rate between the swaddling and sedation groups $(12.5 \%[n=4]$ vs. $4.3 \%[\mathrm{n}=2], P=0.174)$. The average MRI scan time was longer in the swaddling group than in the sedation group $(76.5 \pm 20.3$ minutes vs. $61.5 \pm 13.6$ minutes, $P=0.001)$. Most patients who had apnea or desaturation at the time of MRI examination recovered normal oxygen saturation by taking them out of the MRI cage or providing stimulation, which was also included in the MRI running time. The mean time from the end of scanning to the restart of feeding showed trended towards being lower in the swaddling group, but this finding was not statistically significant (57.9 \pm 48.2 minutes vs. $74.0 \pm 70.2$ minutes, $P=0.233$ ) (Table 3 ).

\section{DISCUSSION}

Based on the findings of this study, we concluded that the feed and swaddle technique can be considered before sedation as a preparation method for brain MRI in VLBWIs. This study was conducted during a QI implementation project for MRI methods in newborns at Ajou University Hospital. Therefore, the inclusion criteria for the swaddling and sedation groups were the same (i.e., BW of less than 1,500.0 g), and the patient group was assigned naturally based on the patient's date of birth. In the same context, brain MRI was conducted in patients with a medically stable condition in both groups.

We found that the feed and swaddle technique was an effective method for preparing neonates for brain MRI and should be considered first before administering a sedation protocol. A sedation protocol has been used in pediatric patients to reduce the test time and misdiagnoses because of poor image quality, which may be caused by patient motion artifacts during MRI. However, the sedation method for MRI may cause serious adverse effects in neonates. The most common adverse effect of sedation is respiratory depression caused by apnea, hypopnea, or hypoxia $^{19)}$. Our study also demonstrated that cardiopulmonary adverse events were significantly higher in the sedation group
( $P=0.002)$ (Table 3$)$. In addition, previous studies have shown that certain anesthetics and sedatives may have a detrimental effect on the developing brain in animal models ${ }^{20,21)}$. No conclusive evidence has shown that the same effect occurs in humans, although studies have shown that children under 3 years of age who are exposed to anesthesia may have impaired learning ${ }^{20,21)}$. Another disadvantage of sedatives is the limited number of sedatives that are safe in preterm infants. Therefore, to reduce the risks associated with anesthesia and sedation during the imaging of newborns, non-pharmacological interventions have been studied to replace the sedation protocol.

In the swaddling method, images are taken during natural sleep immediately after feeding, and attention is focused on processes such as noise and light reduction and warming to minimize movement. The MRI failure rate was $12.5 \%(n=4)$ in the swaddling group, which was higher than the failure rate of $4.3 \%(n=2)$ in the sedation group. However, this finding was not statistically significant. In many studies, the use of the MedVac vacuum splint for non-sedated neonates has an MRI success rate of $7 \%$ to $100 \%^{15,22-26)}$. The failure rate of MRI when using the swaddling technique in our center was comparable to that reported by other centers ${ }^{4,15,27)}$. Several other studies reported that the MRI failure rate was higher in the feed and swaddle groups, but the preparation time was significantly shorter than that of the sedation group ${ }^{27,28)}$.

The MRI scan time was significantly longer in the swaddling group than in the sedation group $(76.5 \pm 20.3$ minutes vs. $61.5 \pm$ 13.6 minutes, $P=0.001$ ). This finding may be due to frequent movement during MRI scans among the swaddling group pati ents. Swaddling increases the scan time; however, the preparation time for MRI is less; therefore, the overall MRI time would be comparable between the two groups ${ }^{27)}$. In addition, the scan time in infants, which is longer than that in adults, includes the process of adjusting oxygen saturation or heart rate change on the monitor and frequent movements that may occur in newborns. This study also demonstrated that the burden of patient monitoring was reduced in the swaddling group. The bedside effort of the physician and physician's assistant decreased throughout the imaging process, although this effort was not quantified.

A disadvantage of the swaddling method is that the scan time can be lengthened if the test has to be repeated because of poor image quality or the patient's inability to resume sleep. However, despite the potential for delays, several studies have reported that imaging neonates without anesthesia and sedation can improve 
the imaging process, reduce costs, and improve resource utiliza$\operatorname{tion}^{15,24,26)}$.

This study has several limitations. The first limitation is its retrospective design and the possibility of selection bias. However, based on the background of this study with regard to QI, this study was conducted over a sufficient period and in a sufficiently large population in the context of policy changes in which brain MRI was conducted for all neonates with a BW of less than 1,500 g. In addition, this study was not prospective; however, we found no difference in GA, BW, and morbidity between the compared groups during the study period. Thus, this study's findings are meaningful, and the possibility of selection bias can be compensated for. Second, this study was not a randomized controlled trial; therefore, stating that the results are strong is difficult. Well-designed randomized controlled trials are required in the future.

In conclusion, when conducting brain MRI in VLBWIs, the swaddling method rather than sedation should be considered to reduce cardiopulmonary adverse events. In addition, particularly in newborns, anesthesia and sedation should be avoided, unless the response to painful stimuli is necessary for evaluation.

In conclusion, the success rate of MRI was comparable between the swaddling technique and sedation in premature infants. Furthermore, despite the drawback of prolonged scan time, swaddling can reduce the rate of cardiopulmonary adverse events relative to that with the use of sedative agents. Therefore, the feed and swaddling technique during routine brain MRI may be worth attempting in an NICU to avoid sedation or anesthesia.

\section{ARTICLE INFORMATION}

\section{Ethical statement}

This study was approved by the Institutional Review Board (IRB) for waivers of all forms of informed consent (IRB number: MED-MDB-19-388).

\section{Conflicts of interest}

No potential conflict of interest relevant to this article was reported.

\section{Author contributions}

Conception or design: Y.M.Y., M.S.P., J.H.L.

Acquisition, analysis, or interpretation of data: Y.M.Y., J.E.P.,
M.S.P., J.H.L.

Drafting the work or revising: Y.M.Y., M.S.P., J.H.L.

Final approval of the manuscript: Y.M.Y., M.S.P., J.H.L.

\section{ORCID}

Yeong Myong Yoo https://orcid.org/0000-0001-9429-8474

Jang Hoon Lee https://orcid.org/0000-0003-4765-9948

\section{Acknowledgments}

None

\section{REFERENCES}

1. Blencowe H, Lee AC, Cousens S, Bahalim A, Narwal R, Zhong $\mathrm{N}$, et al. Preterm birth-associated neurodevelopmental impairment estimates at regional and global levels for 2010. Pediatr Res 2013;74 Suppl 1:17-34.

2. Sutton PS, DarmstadtGL.Preterm birth and neurodevelopment: a review of outcomes and recommendations for early identification and cost-effective interventions. J Trop Pediatr 2013; 59:258-65.

3. Banihani R, Seesahai J, Asztalos E, Terrien Church P. Neuro imaging at term equivalent age: is there value for the preterm infant? A narrative summary. Children (Basel) 2021;8: 227.

4. Antonov NK, Ruzal-Shapiro CB, Morel KD, Millar WS, Kashyap S, Lauren CT, et al. Feed and wrap MRI technique in infants. Clin Pediatr (Phila) 2017;56:1095-103.

5. Eker HE, Cok OY, Cetinkaya B, Aribogan A. Oral 30\% glucose provides sufficient sedation in newborns during MRI. J Anesth 2017;31:206-11.

6. Parad RB. Non-sedation of the neonate for radiologic proce dures. Pediatr Radiol 2018;48:524-30.

7. Boutros A, Pavlicek W. Anesthesia for magnetic resonance imaging. Anesth Analg 1987;66:367.

8. Hupp JR. Pediatric anesthesia: concerns about neurotoxicity. J Oral Maxillofac Surg 2015;73:1021-2.

9. Durrmeyer X, Vutskits L, Anand KJ, Rimensberger PC. Use of analgesic and sedative drugs in the NICU: integrating clinical trials and laboratory data. Pediatr Res 2010;67:117-27.

10. Sun L. Early childhood general anaesthesia exposure and neurocognitive development. Br J Anaesth 2010;105 Suppl 1: i61-8.

11. Sprung J, Flick RP, Katusic SK, Colligan RC, Barbaresi WJ, Bojanic K, et al. Attention-deficit/hyperactivity disorder after early exposure to procedures requiring general anesthesia. Mayo Clin Proc 2012;87:120-9.

12. DiMaggio C, Sun LS, Li G. Early childhood exposure to 
anesthesia and risk of developmental and behavioral disorders in a sibling birth cohort. Anesth Analg 2011;113:1143-51.

13. Cote CJ, Karl HW, Notterman DA, Weinberg JA, McCloskey C. Adverse sedation events in pediatrics: analysis of medications used for sedation. Pediatrics 2000;106:633-44.

14. Havidich JE, Beach M, Dierdorf SF, Onega T, Suresh G, Cravero JP. Preterm versus term children: analysis of sedation/anesthe sia adverse events and longitudinal risk. Pediatrics 2016;137: e20150463.

15. Haney B, Reavey D, Atchison L, Poull J, Dryer L, Anderson B, et al. Magnetic resonance imaging studies without sedation in the neonatal intensive care unit: safe and efficient. J Perinat Neonatal Nurs 2010;24:256-66.

16. Ureta-Velasco N, Martinez-de Aragon A, Moral-Pumarega MT, Nunez-Enamorado N, Bergon-Sendin E, Pallas-Alonso CR. Magnetic resonance imaging without sedation in neonates. An Pediatr (Barc) 2015;82:354-9.

17. Finnemore A, Toulmin H, Merchant N, Arichi T, Tusor N, Cox D, et al. Chloral hydrate sedation for magnetic resonance imaging in newborn infants. Paediatr Anaesth 2014;24:190-5.

18. Executive summary: neonatal encephalopathy and neurologic outcome, second edition. Report of the American College of Obstetricians and Gynecologists' Task Force on Neonatal Encephalopathy. Obstet Gynecol 2014;123:896-901.

19. Cote CJ, Wilson S; American Academy of Pediatrics; American Academy of Pediatric Dentistry. Guidelines for monitoring and management of pediatric patients before, during, and after sedation for diagnostic and therapeutic procedures: update 2016. Pediatrics 2016;138:e20161212.

20. Davidson AJ, Becke K, de Graaff J, Giribaldi G, Habre W, Hansen $\mathrm{T}$, et al. Anesthesia and the developing brain: a way forward for clinical research. Paediatr Anaesth 2015;25:447-52.

21. Gleich SJ, Flick R, Hu D, Zaccariello MJ, Colligan RC, Katusic SK, et al. Neurodevelopment of children exposed to anesthesia: design of the Mayo Anesthesia Safety in Kids (MASK) study. Contemp Clin Trials 2015;41:45-54.

22. Fogel MA, Pawlowski TW, Harris MA, Whitehead KK, Keller MS, Wilson J, et al. Comparison and usefulness of cardiac magnetic resonance versus computed tomography in infants six months of age or younger with aortic arch anomalies without deep sedation or anesthesia. Am J Cardiol 2011;108:120-5.

23. Golan A, Marco R, Raz H, Shany E. Imaging in the newborn: infant immobilizer obviates the need for anesthesia. Isr Med Assoc J 2011;13:663-5.

24. Mathur AM, Neil JJ, McKinstry RC, Inder TE. Transport, monitoring, and successful brain MR imaging in unsedated neonates. Pediatr Radiol 2008;38:260-4.

25. Shariat M, Mertens L, Seed M, Grosse-Wortmann L, Golding F, Mercer-Rosa L, et al. Utility of feed-and-sleep cardiovascular magnetic resonance in young infants with complex cardiovascular disease. Pediatr Cardiol 2015;36:809-12.

26. Windram J, Grosse-Wortmann L, Shariat M, Greer ML, Crawford MW, Yoo SJ. Cardiovascular MRI without sedation or general anesthesia using a feed-and-sleep technique in neonates and infants. Pediatr Radiol 2012;42:183-7.

27. Yamamura K, Takatsu Y, Miyati T, Inatomi T. Brain magnetic resonance imaging using a customized vacuum shape-keeping immobilizer without sedation in preterm infants. Magn Reson Imaging 2018;54:171-5.

28. Heller BJ, Yudkowitz FS, Lipson S. Can we reduce anesthesia exposure? Neonatal brain MRI: swaddling vs. sedation, a national survey. J Clin Anesth 2017;38:119-22. 